\title{
ÉQUILIBRE ACIDO-BASIQUE DU SANG ET COMPOSITION IONIQUE DU PLASMA CHEZ LA POULE EN HYPERCAPNIE CHRONIQUE
}

\author{
P. MONGIN et B. SAUVEUR \\ avec la collaboration technique de Denise DuBors \\ Station de Recherches avicoles, \\ Centre de Recherches de Tours, 37 - Nouzilly (France) \\ Institut national de la Recherche agronomique
}

\section{INTRODUCTION}

Il a été montré par Mongin et LACASSAGNe (I966 $a$ et b) que la formation de la coquille de l'cuf affecte 1'équilibre acido-basique sanguin de la poule pondeuse dans le sens d'une acidose métabolique partiellement compensée ; l'hydratation de 1'albumen qui se produit après l'entrée de l'œuf dans l'utérus dépend de ce même équilibre puisque la chute de la bicarbémie apparaît dès cet instant. De plus, des acidoses métaboliques d'origine externe modifient simultanément la formation de la coquille et celle de l'albumen (Hali, et Hzibacka, i959; Hun't et Artren, I962 ; SaUveur, I970). I1 est également possible de déplacer l'équilibre acido-basique des animaux par augmentation de la teneur en $\mathrm{CO}_{2}$ de l'atmosphère ; c'est ce qu'ont fait HELBACKA et al. (1963) et Srmkiss (1967) pour étudier respectivement les effets d'une hypercapnie aiguë sur la qualité de l'œuf et les variations de pouvoir tampon du sang qui en résultent. Les expériences de Franck et BurGri (I966) et celles réalisées dans notre laboratoire ont été basées sur l'utilisation du même procédé pendant de plus longues périodes; nous avons constaté en particulier qu'il était possible par ce moyen de modifier de façon durable la solidité de la coquille et la qualité de l'albumen (Mongin, I968; SAUveur, Ig68).

Le présent rapport a pour objet de décrire les modifications des équilibres acidobasique et ionique du sang observées au cours d'une telle hypercapnie chronique; il démontre que la réponse sanguine de la poule à une surcharge prolongée en $\mathrm{CO}_{2}$ est très proche de celles déjà observées chez les Mammifères. 


\section{MATÉRIEL, ET MÉTHODES}

\section{Animaux}

Les mesures ont été effectuées sur 96 poules pondeuses dont 48 de race Leghorn et 48 issues d'un croisement Rhode Island Red $\times$ Wyandotte âgées de 13 mois. Ces animaux ont reçu pendant toute la durée du traitement un aliment "reproducteur " standard et 16 heures de lumière par jour.

\section{Réalisation de l'hypcrcapnie}

Les animaux ont été répartis au hasard en 2 lots et placés pendant trois mois dans des poulaillers conditionnés dont les caractéristiques étaient, pour le premier : température $20^{\circ} \mathrm{C}$, humidité relative 65 p. Ioo, teneur de l'air en $\mathrm{CO}_{2} 3$ p. Ioo contrôlée automatiquement par un analyseur à infrarouge (Contrôle de Chauffe, Licence Onera). Le second poulailler (témoin) était identique exception faite de la tencur normale de l'air en $\mathrm{CO}_{2}(0,03$ p. IOO).

\section{Prélèvements de sang et mesures}

Durant l'heure suivant une oviposition, nous avons prélevé au moyen de seringues hépari nées, un échantillon de sang dans la veine alaire gauche de chaque animal; nous avons cnsuite mesuré le pl et la $\mathrm{pCO}_{2}$ du sang ainsi que les teneurs en sodium,potassium, calcium, chlore. (". phosphore total du plasma suivant cles méthodes déjà décrites (SAUVEUR, I969).

\section{RÉSULTATS}

\section{r. Équilibre acido-basique du sang}

Nous avons reporté au tableau I les moyennes des valeurs de $\mathrm{pH}, \mathrm{pCO}_{2}$ et bicarbémie des animaux témoins et de ceux soumis à la surcharge en $\mathrm{CO}_{2}$; pour chaque paramètre, la première ligne de résultats est relative aux poules de race Leghorn et la seconde aux $R \times W$; la troisième ligne représente la moyenne de toutes les observations.

Nous enregistrons sous l'action de l'hypercapnie chronique un léger abaissement du $\mathrm{pH}$ sanguin $(-0,03 ; p<0,05)$ et un accroissement très net de la $\mathrm{pCO}_{2}$ $(+\mathrm{I} 4, \mathrm{I} \mathrm{mm} \mathrm{Hg})$ et de la teneur en bicarbonates $(+6,8 \mathrm{méq} / 1)(p<0,0005$ pour ces 2 valeurs). Ces résultats sont valables pour l'ensemble des animaux mais il semble que les réponses des Leghorn sont supérieures à celles des $R \times W$; c'est ainsi que pour la $\mathrm{pCO}_{2}$ la différence " $\mathrm{CO}_{2}$ " - témoin est de $\mathrm{I} 5,7$ chez les premières contre $\mathrm{I} 2,5$ chez les secondes.

Remarquons enfin que les élévations relatives moyennes de $\mathrm{pCO}_{2}$ et de bicarbémie dues à l'hypercapnie chronique sont tout à fait comparables ( 29 et 28,9 p. Ioo respectivement) ce qui explique le très faible abaissement de $\mathrm{pH}$ qui ne représente que 0,3 p. roo de la valeur témoin.

L'adaptation de l'équilibre acido-basique à la surcharge prolongée en $\mathrm{CO}_{2}$ peut également être exprimée par la régression de la teneur en ions $\mathrm{H}^{+}$du sang sur sa $\mathrm{pCO}_{2}$; ce mode d'expression a l'avantage de faire apparaître une relation linéaire 
entre l'acidité sanguine et la $\mathrm{pCO}_{2}$, ce qui n'est pas possible avec la notation $\mathrm{pH}$. Le calcul, effectué sur l'ensemble de nos données, aboutit au résultat:

$$
\left[\mathrm{H}^{+}\right]=0,324 \mathrm{pCO}_{2}+28,05
$$

avec

$$
r\left[\mathrm{H}^{+}\right], \mathrm{pCO}_{2}=0,67 \quad(\mathrm{~F}=79,8 p<0,0005),
$$

relation dans laquelle $\left[\mathrm{H}^{+}\right]$est exprimée en nanomoles/litre et $\mathrm{pCO}_{2}$ en $\mathrm{mm} \mathrm{Hg}$. $\mathrm{La}$ droite de régression correspondante est représentée sur la figure I; compte tenu du

\section{TABLEAU I}

Action de l'hypercapnie chronique (3 p. Ioo $\mathrm{CO}_{2}$ )

\begin{tabular}{|c|c|c|c|}
\hline & Témoin & Hypercapnie & $\mathrm{F}$ \\
\hline $\mathrm{pH}$ & $\begin{array}{r}\text { L. 7,374 } \pm 0,008(a) \\
\text { R. W. 7,3'1 } \pm 0,011(a) \\
7,358 \pm 0,007(b)\end{array}$ & $\begin{array}{l}7,349 \pm 0,009 \\
7,317 \pm 0,011^{\prime} \\
7,333 \pm 0,008\end{array}$ & $\begin{array}{l}3,72 \\
1,75 \\
4,80\left(^{*}\right)\end{array}$ \\
\hline $\mathrm{pCO}_{2}\left({ }^{1}\right)$ & $\begin{array}{l}14,99 \pm 1,22 \\
49,78 \pm 3,36 \\
\div 7,38 \pm 1,80\end{array}$ & $\begin{array}{l}60,71 \pm 1,49 \\
62,29 \pm 2,58 \\
61,52 \pm 1,68\end{array}$ & $\begin{array}{c}66,74(* * *) \\
8,41(* *) \\
35,78(* * *)\end{array}$ \\
\hline$\left[\mathrm{HCO}_{3}{ }^{-}\right]\left({ }^{2}\right)$ & $\begin{array}{l}2,33 \pm 0,62 \\
22,29 \pm 1,26 \\
23,31 \pm 0,71\end{array}$ & $\begin{array}{l}30,87 \pm 0,81 \\
29,29 \pm 0,80 \\
30,08 \pm 10,37\end{array}$ & $\begin{array}{l}41,08(* * *) \\
24,28(* * *) \\
63,3+(* * *)\end{array}$ \\
\hline
\end{tabular}
sur l'équilibre acido-basique du sang de la poule

(1) en $\min \mathrm{Hg}$.

(अ) en méq/itre.

(a) $: \bar{x}+\frac{\sigma}{\sqrt{n}}$ de 2't mesures.

(b) $: \bar{x}+\frac{\sigma}{\sqrt{n}}$ de 48 mesures.

(*) Significatif au seuil $p<0,05$.

(**) Significatif au seuil $p<0,01$.

$(* * *)$ Significatif au seuil $p<0,001$

caractère linéaire de la relation, nous pouvons calculer les intervalles de confiance de $\left[\mathrm{H}^{+}\right]$pour différentes valeurs de $\mathrm{pCO}_{2}$ : ceux de la figure I ont été choisis au seuil de probabilité 0,05 .

Les concentrations des ions $\mathrm{H}^{+}$et $\mathrm{HCO}_{3}^{-}$et la $\mathrm{pCO}_{2}$ du sang sont liées par la loi d'action de masse suivant une relation de la forme :

$$
\frac{\left[\mathrm{H}^{+}\right]\left[\mathrm{HCO}_{3}^{-}\right]}{\text {a.p } \mathrm{pCO}_{2}}=\mathrm{K}^{\prime}
$$

Nous pouvons donc, à partir des équations (I) et (2) établir la relation directe stuivante entre la bicarbémie et la $\mathrm{pCO}_{2}$ :

$$
\left[\mathrm{HCO}_{3}^{-}\right]=\frac{22,86 \mathrm{pCO}_{2}}{0,324 \mathrm{pCO}_{2}+28,05}
$$


avec $\left[\mathrm{HCO}_{3}^{-}\right]$exprimée en méq $/ 1$ et $\mathrm{pCO}_{2}$ en $\mathrm{mm} \mathrm{Hg}$. La courbe correspondante et ses intervalles de confiance $(p=0,05)$ sont représentés sur la figure 2. Celle-ci, de même que la figure I, caractérise la réponse normale de la poule à une hypercapnie chronique ; pour plus de précision nous rapportons également au tableau 2 les limites exactes des intervalles de confiance calculés.

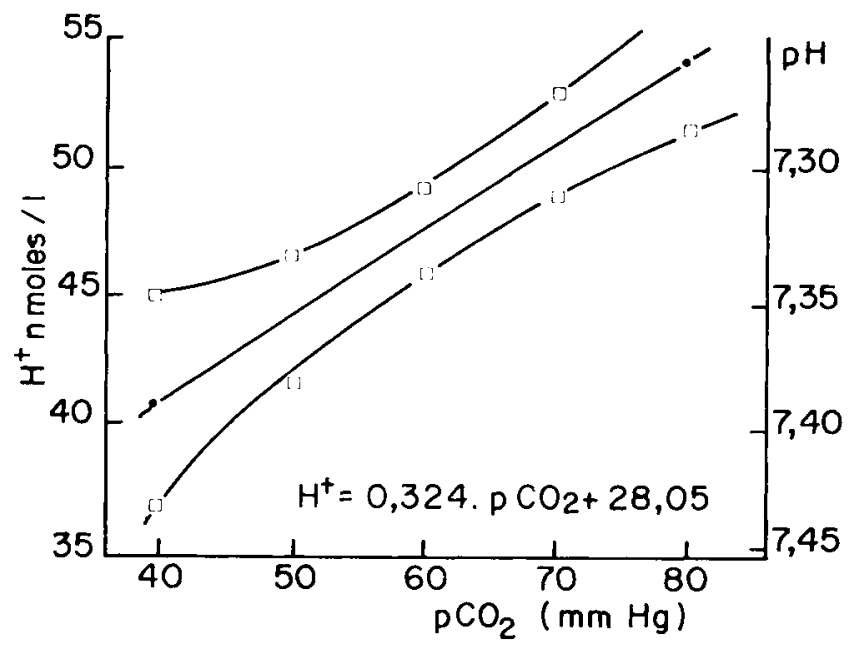

FIG. I. - Relation entre la teneur en protons et la $\mathrm{pCO}_{2}$ du sang veineux général chez la poule en hypercapnie chronique (avec intervalles de confiance au seuil de probabilité 5 p. Ioo)

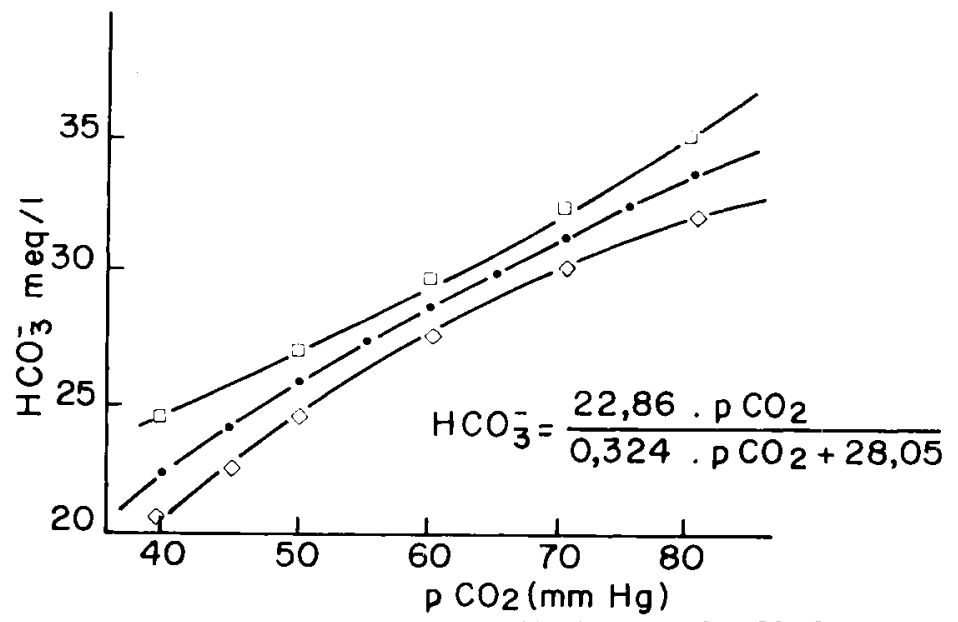

FIG. 2. - Relation entre la teneur en bicarbonates et la $\mathrm{pCO}_{2}$ du sang chez la poule en hypercapnie chronique

(aves intervalles de confiance au seuil de probabilité 5 p. Ioo) 
TABLEAU 2

Intervalles de confiance au seuil de probabilité 0,05

de la teneur en ions hydrogènes et bicarbonates du sang

au cours de l'hypercapnie chronique (3 p. Ioo $\mathrm{CO}_{2}$ )

\begin{tabular}{c|c|c|c|c}
\hline $\mathrm{pCO}_{2}$ & $\begin{array}{c}\text { Nombre } \\
\text { d'observations }\end{array}$ & $\begin{array}{c}{\left[\mathrm{H}^{+}\right]} \\
\text {(nanomoles/litre) }\end{array}$ & $\mathrm{pH}$ & $\begin{array}{c}{\left[\mathrm{HCO}_{3}^{-}\right]} \\
(\mathrm{méq} / \mathrm{l})\end{array}$ \\
\hline & & $37,02-44^{\prime}, 96$ & $7,43-7,35$ & $24,71-20,35$ \\
50 & 6 & $41,96-46,54$ & $7,38-7,33$ & $27,25-24,57$ \\
60 & 16 & $45,73-49,25$ & $7,34-7,31$ & $30,00-27,86$ \\
70 & 30 & $48,71-52,75$ & $7,31-7,28$ & $32,86-30,35$ \\
80 & 28 & $51,25-56,63$ & $7,29-7,25$ & $35,70-32,31$ \\
\hline
\end{tabular}

\section{2. Équilibre ionique du plasma}

Les teneurs plasmatiques en ions sodium, potassium, calcium, chlore et en phosphore total sont reportées au tableau 3. Il y apparait que seule la kaliémie est liée aux variations de l'équilibre acido-basique ; son accroissement n'est d'ailleurs significatif que chez les poules de race Leghorn chez lesquelles il représente 32,7 p. Ioo de la valeur témoin.

La faible augmentation apparente de la natrémie n'atteint pas le seuil de signification statistique de $5 \mathrm{p}$. Ioo et les concentrations des ions $\mathrm{Ca}^{++}$et $\mathrm{Cl}^{-}$et de $\mathrm{P}$ total sont identiques chez les animaux témoins et ceux en hypercapnie.

Compte tenu des variations de kaliémie enregistrées il nous a paru intéressant de calculer le coefficient de corrélation entre la teneur en potassium et la $\mathrm{pCO}_{2}$ plasmatiques ; il apparaît, à partir des moyennes par traitement, que ces deux paramètres sont liés suivant la relation :

avec

$$
\left[\mathrm{K}^{+}\right]=0,085 \mathrm{pCO}_{2}+0,33
$$

$$
r\left[\mathrm{~K}^{+}\right], p \mathrm{CO}_{2}=0,76(p<0,05)
$$

Il semble enfin que la bicarbémie et la chlorémie tendent à varier de façon inverse $(r$ inter-traitements $=0,66$ qui approche le seuil de signification 0,05$)$; nous avons vu cependant que l'abaissement de la chlorémie sous l'effet de l'hypercapnie chronique n'est pas significatif ce qui indique que, dans nos conditions expérimentales, la liaison évoquée ci-dessus doit être faible.

\section{Effet de la race de la pondeuse}

Quel que soit l'état physiologique considéré, il existe pour la plupart des paramètres étudiés des variations d'origine génétique indiquées au tableau 4 . Nous constatons en particulier que les Leghorn ont une calcémie et une bicarbémie supérieures à celles des $R \times W$. Notons également que la phosphorémie est plus élevée chez les Leghorn de même que le $\mathrm{pH}$ sanguin ; ce dernier résultat, joint à la teneur plus grande en bicarbonates, montre que la compensation de l'acidose ventilatoire a été plus développée chez les poules de race Leghorn que chez les $R \times W$. 
TABLEAU 3

Action de l'hypercapnie chronique sur la concentration des principaux éléments minéraux du plasma

$\left(\mathrm{Na}^{+}, \mathrm{K}^{+}, \mathrm{Ca}^{++}\right.$, et $\mathrm{Cl}^{-}$en méq/l; $\mathrm{P}$ en $\left.\mathrm{mg} / \mathrm{l}\right)$

\begin{tabular}{|c|c|c|c|}
\hline & Témoins & Hypercapnic & $\mathbf{F}$ \\
\hline $\mathrm{Na}^{+}$ & $\begin{array}{l}161,81 \pm \quad 6,88(a) \\
163,1 \times \pm 7,10 \\
162,61 \pm \quad 5,00(b)\end{array}$ & $\begin{array}{l}179,56 \pm \quad 8,87 \\
173,66 \pm \quad 9,09 \\
176,61 \pm \quad 6,29\end{array}$ & $\begin{array}{l}2,57 \\
0,72 \\
2,94\end{array}$ \\
\hline $\mathrm{K}^{+}$ & $\begin{array}{l}3,97 \pm 0,21 \\
4,75 \pm 0,36 \\
4,36 \pm 0,21\end{array}$ & $\begin{array}{l}5,27 \pm 0,36 \\
5,4^{\prime} \pm 0,49 \\
5,55 \pm 0,30\end{array}$ & $\begin{array}{c}11,45(* *) \\
3,10 \\
10,7\left(^{* *}\right)\end{array}$ \\
\hline $\mathrm{Ca}^{++}$ & $\begin{array}{l}16,81 \pm 1,19 \\
13,61 \pm 0,72 \\
15,21 \pm 0,73\end{array}$ & $\begin{array}{l}17,99 \pm 0,67 \\
11,31 \pm 1,07 \\
16,17 \pm 0,68\end{array}$ & $\begin{array}{l}0,72 \\
0,31 \\
1,00\end{array}$ \\
\hline $\mathrm{Cl}^{-}$ & $\begin{array}{l}110,71 \pm 1,0)^{\prime} \\
110,96 \pm 1,08 \\
110,83 \pm 0,7 t\end{array}$ & $\begin{array}{l}108,62 \pm 0,95 \\
111,33 \pm 1,05 \\
109,98 \pm 0,73\end{array}$ & $\begin{array}{l}2,33 \\
0,06 \\
0,63\end{array}$ \\
\hline P.. & $\begin{array}{l}495,18 \pm 25,71 \\
439,73 \pm 48,67 \\
467,45 \pm 27,51\end{array}$ & $\begin{array}{l}512,18 \pm 47,06 \\
373,36 \pm 22,23 \\
4,2,77 \pm 27,81\end{array}$ & $\begin{array}{l}0,10 \\
1,50 \\
0,43\end{array}$ \\
\hline
\end{tabular}

(**) Significatif au seuil $p<0,01$.

(a) $: \bar{x}+\frac{\sigma}{\sqrt{n}}$ de 24 inesures.

(b) $: \bar{x}+\frac{\sigma}{\sqrt{n}}$ de 48 mesures.

TABLEAU 4

Effet de la race de la poule sur los paramètres sanguins

\begin{tabular}{|c|c|c|c|}
\hline & Leghorn & Rir $\times \mathrm{I}^{r} y a n d o t t e$ & $\mathrm{~F}$ \\
\hline $\mathrm{Na}^{+}$méq/1 & $170,68 \pm 5,70$ & $168,57 \stackrel{1}{ \pm} 5,85$ & 0,07 \\
\hline $\mathrm{K}^{+} \ldots \ldots$ & $4,62 \pm 0,23$ & $5,29 \pm 0,31$ & 3,44 \\
\hline $\mathrm{Ca}^{++}$ & $17, \dot{\prime} \pm 0,68$ & $13,98 \stackrel{1}{ \pm} 0,6 !$ & $12,78(* * *)$ \\
\hline $\mathrm{Cl}^{-} \ldots \ldots \ldots$ & $109,67 \pm 0,71$ & $111,14 \pm 0,74$ & 2,03 \\
\hline $\mathrm{HCO}_{3}^{-} \ldots \ldots \ldots \ldots \ldots$ & $27,60 \pm 0,69$ & $25,79 \pm 0,90$ & $4,47(*)$ \\
\hline $\mathrm{P} m g / 1 \ldots \ldots \ldots \ldots$ & $503,68 \pm 26,53$ & $400,54 \pm 26,90$ & $6,61\left(^{*}\right)$ \\
\hline $\mathrm{pCO}_{2} \mathrm{~mm} / \mathrm{Hg} \ldots$ & $52,86 \pm 1,49$ & $56,04 \pm 2,29$ & 1,81 \\
\hline $\mathrm{pH} \ldots \ldots \ldots \ldots \ldots \ldots$, & $7,362 \pm 0,006$ & $7,3 \geq 9 \pm 0,009$ & $8,52(* *)$ \\
\hline
\end{tabular}

(*) Significatif au seuil $p<0,05$.

(**) Significatif au seuil $p<0,01$.

$(* * *)$ Significatif au seuil $p<0,001$. 


\section{DISCUSSION}

On sait que chez les Mammifères, la réponse de l'organisme à une surcharge prolongée en $\mathrm{CO}_{2}$ se différencie essentiellement de l'état d'hypercapnie aiguë par l'intervention régulatrice du rein; pendant les 5 à 6 premiers jours de l'exposition au $\mathrm{CO}_{2}$ l'excrétion d'acidité nette et d'ammonium s'accroît considérablement en même temps que sont régénérés des ions bicarbonates. Ceux-ci viennent s'ajouter à ceux libérés par les tampons corporels dès le début de la surcharge en $\mathrm{CO}_{2}$, assurant ainsi la lutte contre l'élévation du $\mathrm{pH}$ extracellulaire. Passé ce délai, l'acidurie revient à la normale tandis que la bicarbémie, cessant de croître, se maintient à un niveau très supérieur à la normale; cette situation, caractéristique de l'état d'hypercapnie chronique, a été décrite en détail chez le Chien par Schwartz et al. (I965) et chez l'Homme (VAN YPERSELE de STRIHOU et al., I966), l'ensemble des travaux ayant été résumé par VAN YPERSEI E de Strihou et Frans (I967). Nous avons voulu quant à nous étudier les réponses sanguines de la Poule à de tels traitements qui se sont par ailleurs révélés intéressants pour la qualité de la coquille de l'œuf (FRANCK et BuRGER, Ig66; MONGIN, I968).

Lors de leurs travaux sır le Chien, Schwar'Tz et al. (I965) constatent que, malgré l'accroissement progressif de la bicarbémie qui suit les élévations de $\mathrm{pCO}_{2}$, le $\mathrm{pH}$ sanguin ne se rétablit jamais exactement à la valeur normale mais varie avec la $\mathrm{pCO}_{2}$; si l'acidité sanguine est exprimée en nanomôles d'ions $\mathrm{H}^{+} /$litre, la relation est la suivante: $\left[\mathrm{H}^{+}\right]=0,32 \mathrm{pCO}_{2}+26,9$.

Le protocole expérimental utilisé par l'équipe de ScHWARTz est assez éloigné de celui mis en œuvre ici-même puisque les teneurs en $\mathrm{CO}_{2}$ atmosphériques utilisées sont successivement de 7 , II et I 7 p. Ioo contre 3 p. Ioo constant dans la présente expérience. Nous constatons cependant qu'il existe une analogie remarquable entre les deux réponses du chien et de la poule à l'hypercapnie chronique puisque l'équation calculée d'après nos données est la suivante : $\left[\mathrm{H}^{+}\right]=0,324 \mathrm{pCO}_{2}+28,05$

Chez ces deux espèces une élévation de $\mathrm{pCO}_{2}$ de $\mathrm{I} \mathrm{mm} \mathrm{Hg}$ entraîne donc un accroissement de la teneur du sang en ions $\mathrm{H}^{+}$égal à $0,32 \mathrm{n}$. môles/litre. Notons d'ailleurs que l'hypercapnie chronique due à des insuffisances pulmonaires se traduit chez 1'homme par une élévation de l'acidémie à peu près identique : $\left[\mathrm{H}^{+}\right]=0,30 \mathrm{pCO}_{2}+26,8$ (VAN YPERSEI,E de STRIhou et al., I966). Les droites de régression correspondant à ces 3 relations sont juxtaposées sur la figure 3 pour montrer la similitude des réponses d'une espèce à l'autre.

On sait par ailleurs que, simultanément à l'élévation de la bicarbémie, 1'hypercapnie chronique provoque un abaissement de la chlorémie dû vraisemblablement à une excrétion urinaire accrue de chlore (Polak et al., ig6r ; Schwartz et al., I965). Cette manifestation secondaire de la surcharge en $\mathrm{CO}_{2}$ n'apparaît que très peu ici puisque les valeurs de la chlorémie sont identiques chez le lot témoin et celui soumis à une surcharge de $3 \mathrm{p}$. Ioo $\mathrm{CO}_{2}$, ce qui interdit d'accorder beaucoup d'importance au coefficient de corrélation inter-traitement $r \mathrm{Cl}^{-}, \mathrm{HCO}_{3}^{-}$trouvé égal à - o,66; ce dernier n'apparaît d'ailleurs pas significatif. Remarquons cependant que la durée du traitement appliqué ( 3 mois) est très supérieure à celles utilisées par les auteurs pré- 
cédents et qu'un nouvel état d'équilibre a pu s'établir. Il semble en effet que l'espèce utilisée ne soit pas directement en cause puisque, lors d'expériences de nature différente, nous avons pu noter chez la poule l'existence d'une relation inverse entre les teneurs en chlore et en bicarbonates du sang (Mongrn, I970; SAUveur, I969). Seule une étude approfondie de l'excrétion urinaire des électrolytes après de longues périodes d'hypercapnie pourrait aider à la compréhension des phénomènes enregistrés jci.

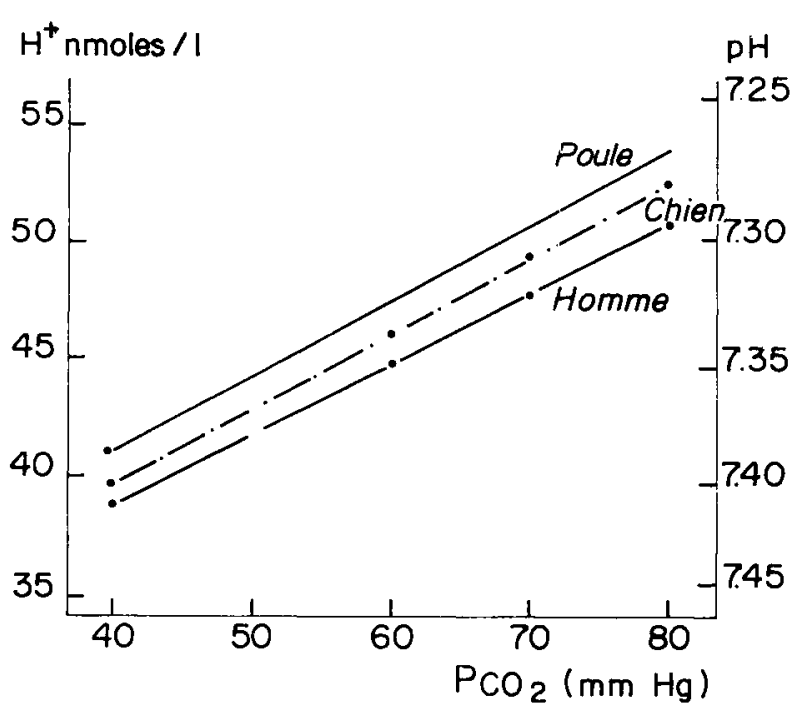

FIG. 3. - Relation $\left[\mathrm{H}^{+}\right]-p \mathrm{CO}_{2}$ durant une hypercapnie chronique chez la Poule, le Chien et l'Homme

Les valeurs normales de la calcémie et de la phosphorémie observées chez nos animaux en hypercapnie s'accordent avec les résultats de SCHAFFFER et al. (I963) qui notent chez l'Homme un retour à la normale de ces deux paramètres après 23 jours d'exposition à $I, 5$ p. Ioo de $\mathrm{CO}_{2}$; les auteurs supposent qu'après cette période, un nouvel équilibre calcique est atteint entre le sang et l'os tandis que la valeur normale de la phosphorémie peut, à ce stade, être attribuée au caractère essentiellement " compensé " de l'acidose respiratoire.

L'accroissement de la kaliémie que nous rapportons et qui semble être lié à l'élévation de $\mathrm{pCO}_{2}$ a déjà été décrit chez des chiens en " rebreathing " par LADE et BRown (I963) mais pas, à notre connaissance, lors d'hypercapnies chroniques.

Rappelons, pour terminer, que nous observons des variations des composantes sanguines attribuables à la race des pondeuses. Ainsi les teneurs en calcium, phosphore et bicarbonate sont plus élevées chez les Leghorns que chez les $R I R \times W y$ andottes; or les études comparatives que nous effectuons depuis plusieurs années sur la solidité des coquilles des oufs de chacune de ces 2 races (résultats non publiés) montrent une nette supériorité de la première sur la seconde qui pourrait être en partie due aux variations de constantes sanguines ci-dessus.

En conclusion, si l'élévation de $\mathrm{pCO}_{2}$ sanguine entraîne chez la poule en hypercapnie chronique, un accroissement des teneurs en ions $\mathrm{H}^{+}$et $\mathrm{HCO}_{3}^{-}$du sang abso- 
lument identique à ceux déjà décrits chez le Chien et l'Homme, il semble à l'opposé que les variations des électrolytes plasmatiques soient quelque peu différentes et justiciables d'études plus approfondies.

\title{
RÉSUMÉ
}

Nous avons étudié les effets d'une hypercapnie chronique sur les équilibres acido-basique et électrolytique du sang chez 48 poules placées pendant trois mois dans une atmosphère contenant $3 \mathrm{p}$. Ioo de $\mathrm{CO}_{2} ; 48$ autres animaux élevés en atmosphère normale ont servi de témoins. Dans chacun des deux lots nous disposions pour moitié de poules de race Leghorn et d'autres issues d'un croisement Rhode Island $\times$ Wyandotte. Les résultats portent d'une part sur le $\mathrm{pH}$, la $\mathrm{pCO}_{2}$ et la bicarbémic et d'autre part sur la teneur en ions $\mathrm{Na}^{+}, \mathrm{K}^{+}, \mathrm{Ca}^{++}, \mathrm{Cl}^{-}$et en phosphore total du plasma enregistrés en fin d'expérience.

L'état d'hypercapnie chronique se caractérise par une forte élévation de la $\mathrm{pCO}_{2}$ sanguine $\left(+\mathrm{I}_{4}, \mathrm{I} \mathrm{mm} \mathrm{Hg}\right)$ et de bicarbémie $(+6,8 \mathrm{méq} / \mathrm{l})$ accompagnée d'un léger abaissement du $\mathrm{pH}$ (- 0,03 unité $\mathrm{pH}$ ). Nous étudions plus particulièrement les variations des teneurs en ions $\mathrm{H}^{+}$ et $\mathrm{HCO}_{3}$ - en fonction de la $\mathrm{pCO}_{2}$ du sang qui chiffrent de façon précise la réponse normale de la poule à une surcharge prolongée en $\mathrm{CO}_{2}$. La comparaison de ces résultats avec ceux obtenus chez les Mammifères montre que la fonction $\left[\mathrm{H}^{+}\right]=f\left(\mathrm{pCO}_{2}\right)$ est identique chez la Poule, le Chien et l'Homme.

Parmi les électrolytes du plasma, seul le potassium semble être affecté par l'état d'hypercapnie ; en outre, nous notons, pour le $\mathrm{pH}, \mathrm{HCO}_{3}^{-}, \mathrm{Ca}^{++}$et $\mathrm{P}$ total, des variations d'origine génétique.

\section{SUMMARY}

\author{
ACID-BASE AND ELECTROLYTE EQUILIBRIUM \\ DURING CHRONIC HYPERCAPNIA IN THE LAYING HEN
}

We have studied the effects of chronic hypercapnia on plasma electrolytes and acid-base equilibrium in 48 laying hens after three months of exposure to 3 p. 1 oo $\mathrm{CO}_{2} ; 48$ other animals kept in normal atmosphere were used as controls. The studied parameters are blood $\mathrm{pH}^{\mathrm{pCO}} \mathrm{p}$ and bicarbemia and, on another hand, plasma concentrations of $\mathrm{Na}, \mathrm{K}, \mathrm{Ca}, \mathrm{Cl}$ and total $\mathrm{P}$.

The steady state of chronic hypercapnia is characterized by a large increase of blood $\mathrm{pCO}_{2}$ $(+$ I 4. I $\mathrm{mm} \mathrm{Hg}$ ) and bicarbemia $(+6,8 \mathrm{méq} / 1)$ with a small decrease of $\mathrm{pH}(-0.03$ unit). We more particularly study $\mathrm{H}^{+}$and $\mathrm{HCO}_{3}-$ ions levels in relation to blood $\mathrm{pCO}_{2}$; these variations characterize the normal hen's response to long $\mathrm{CO}_{2}$ exposure. The comparison of these results with those obtained in mammals shows that the relation $\left[\mathrm{H}^{+}\right]=f\left(\mathrm{pCO}_{2}\right)$ is similar in hen, dog and man.

Among plasma electrolytes, potassium concentration only seems to be modified by hyper. capnia; on another hand, we observe genetical variations for $\mathrm{pH}, \mathrm{HCO}_{3}{ }^{-}, \mathrm{Ca}$ and total $\mathrm{P}$.

\section{RÉFÉRENCES BIBLIOGRAPHIQUES}

Franck F. R., Burger R. E., 1965. The effect of carbon dioxide inhalation and sodium bicarbonate ingestion on egg shell deposition. Poultry Sci., 44, I604-1606.

Hall K. N., Helbacka N. V., i959. Improving albumen quality. Poultry Sci., 38, III-II4.

Helbacka N. V., Casterline J. L., Smith C. J., 1963. The effect of hight $\mathrm{CO}_{2}$ atmosphere on the laying hen. Poultry Sci., 42, I082-I084.

Hunt J. R., Aitken J. R., 1962. The effect of ammonium and chloride ions in the diet of hens on eggr shell quality. Poultry Sci., 41, 434-438. 
Lade R. I., Brown E. B., 1963. flasma potassium changes during rebreathing in the dog. Proc. Soc. exper. Biol. Med., 112, 330-331.

Mongrn P., I968. Élevage de pondeuses sous atmosphère enrichie en gaz carbonique. Action sur la ponte et la qualité de la coquille. III Congrès Européen d'Aviculture. Jérusalem. Work Group n $n^{\circ}$, p. 5 (abstr.).

MongrN P., I970. Modification de l'excrétion urinaire induite par une perfusion d'acide chez la poule Ann. Biol. anim. Biochim. Biophys., a paraître.

Mongin P., Lacassagne L., I966a. Équilibre acido-basique du sang et physiologic de la formation de la coquille de I'ouf. Ann. Biol. anim. Biochim. Biophys., 6, 93-100.

Mongin P., Lacassagne L., Ig66 $b$. Rythme respiratoire et physiologie de la formation de la coquille de l'œuf. Ann. Biol. anim. Biochim. Biophys., 6, ror-III.

Polak A., Haynie G. D., Hays R. M., Schwartz W. B., ig6r. Effects of chronic hypercapnia on electrolyte and acid-base equilibrium. I. Adaptation. J. Clin. Invest., 40, 1223-1237.

Sauveur B., I968. Élevage de pondeuses sous atmosphère enrichie en gaz carbonique. Action sur la qualité de l'albumen. III Congrès Européen d'Aviculture. Jérusalem. Work Group nº 7 , p. 5 (abstr.)

Sauveur 13., 1969. Acidoses métaboliques expérimentales chez la poule pondeuse. I. Action sur l'équilibre acido-basique du sang et l'excrétion rénale des électrolytes. Ann. Biol, anim. Biochim. Biophys. 9 (3), 379-39r.

Sauvevr L., I970. Acidoses métaboliques expérimentales chez la poule pondeuse. II. Action sur la composition minérale de l'albumen de l'œuf. Ann. Biol. Biochim. Biophys., 10 (1), 81-1oo.

Schaeffer K. E., Nichols G., CAREY C. R., I963. Calcium metabolism in man during acclimatization to carbon dioxide. J. appl. Physiol, 18, 1070-1084.

Schwartz W. B., Brackett N. C., Cohes J. J., ig65. The response of extracellular hydrogen ion concentration to graded degrees of chronic hypercapnia : the physiologic limits of the defense of pH. J. Clin. Invest., 44, 29I-3or.

Simkiss K., I967. Acute hypercapnia and eggshell formation. Nature. 214, 84-86.

VAN YPERSELE DE STRIHOU C., BRASSEUR L., DE CONINCK J., Ig66. The "carbon dioxide response curven for chronic hypercapnia in man, New Engl. J. Med., 2\%5, II7.

VAN YPERSELE DE STRHOU C., FRANS A., I967. L'équilibre acidobasique. Sa terminologie, sa régulation, son interprétation. In "Actualités nephrol. Hôp. Necker", 5-23, Ed. méd. Flammarion, Paris. 